\title{
DUTPase/DPD Inhibitor TAS-114
}

National Cancer Institute

\section{Source}

National Cancer Institute. dUTPase/DPD Inhibitor TAS-114. NCI Thesaurus. Code C116885.

An orally bioavailable inhibitor of both deoxyuridine triphosphatase (dUT Pase) and dihydropyrimidine dehydrogenase (DPD), with potential antineoplastic adjuvant activity. Upon oral administration in combination with a prodrug of the pyrimidine antagonist 5fluorouracil (5-FU), TAS-114 inhibits (DPD), the liver enzyme responsible for rapid catabolism of 5-FU into inactive metabolites. This prevents first-pass metabolism of 5FU, allowing oral administration of the 5-FU prodrug and increasing the efficacy of 5-FU. In addition, as a dUT Pase inhibitor, TAS-114 enhances the antitumor activity of 5-FU by preventing the hydrolysis and breakdown of 5-fluoro-deoxyuridine triphosphate (FdUTP) and deoxyuridine triphosphate (dUTP), which are active metabolites of 5-FU. This promotes DNA polymerase-dependent incorporation of these antimetabolites into DNA and leads to DNA damage and tumor cell death. Co-administration with TAS-114 allows lower dosing of 5-FU prodrugs, which decreases 5-FU-related toxicity, while maintaining therapeutic levels of 5-FU at the tumor site. 